\title{
John Willoughby Layard (1891- 1974). Antropólogo y psicoanalista haciendo etnografía en Melanesia antes de Malinowski ${ }^{1}$
}

John Willoughby Layard (1891-1974). Anthropologist and psychoanalyst who did fieldwork in Melanesia before Malinowski

Fecha de recepción: 10/08/2020

Fecha de aceptación: 18/03/2021

Fecha de publicación: 25/08/2021

https://doi.org/10.48102/if.2021.v1.n2.190

\author{
Leif Korsbaek* \\ leifkorsbaek1941@gmail.com \\ ORCID: https://orcid.org/0000-0003-0247-4499 \\ Doctor en Ciencias Antropológicas \\ Escuela Nacional de Antropología e Historia \\ México
}

\section{Resumen}

El texto es una somera presentación de hechos relevantes de la vida y la obra del antropólogo británico John Willoughby Layard, con cierto énfasis en su trabajo de campo, desarrollado un poco antes que el de Malinowski, también en Melanesia pero en otras islas. Se hace hincapié en su particular situación en la historia de la antropología británica, tanto como seguidor de W. H. R. Rivers - prácticamente el último difusionista con intereses psi-

1 Agradezco la revisión hecha por Marcela Barrión Luna; ella merece una parte del crédito por la publicación.

* Antropólogo social de la Universidad de Copenhague, Dinamarca. Doctor en Ciencias Antropológicas por la Universidad Autónoma Metropolitana, Ciudad de México. Profesor de tiempo completo del Posgrado en Antropología Social de la Escuela Nacional de Antropología e Historia (ENAH) de México. 
coanalíticos - como estudiante y paciente de C. G. Jung. Asimismo, Layard se destaca por ser poco conocido y siempre marginado, como antropólogo y psicoanalista.

\section{Palabras clave}

John Willoughby Layard, antropología británica, trabajo de campo, psicoanálisis, Carl Gustav Jung.

\section{Abstract}

This text is a brief presentation of relevant facts of the life and work of the British anthropologist John Willoughby Layard, with some emphasis on his fieldwork, developed a little before Malinowski's, also in Melanesia but in other islands. There is an important consideration on his unique position in the history of British anthropology, both as a follower of W. H. R. Rivers-practically the last diffusionist with psychoanalytic interests-and as a student and patient of C. G. Jung. Likewise, Layard stands out for being little known and always marginalized, both as an anthropologist and psychoanalyst.

\section{Keywords}

John Willoughby Layard, British anthropology, fieldwork, psychoanalysis, Carl Gustav Jung.

\section{Introducción}

El último estudiante a tratar antes de llegar al final del proceso, la etnografía de Malinowski, ${ }^{2}$ es John Willoughby Layard, a diferencia de aquél un auténtico británico: "John Willoughby Layard, psicólogo analítico y con cierta razón el primer trabajador de campo intensivo en la antropología social británica, falleció en Oxford el 26 de noviembre de 1974", escribió Ian Langham en su obituario (Langham, 1974, p. 237).

Layard nació en 1891 en el sur de Inglaterra y, después de pasar un año viajando en Europa estudiando francés y alemán, se inscribió en Cambridge en 1909, en donde estudió lenguas modernas en el King's College.

2 El presente artículo es parte de un proyecto de escribir un libro acerca de los antropólogos británicos que trabajaron en Melanesia al mismo tiempo que Malinowski y que participaron en la revolución metodológica de la antropología británica: Haddon, Rivers, Selñigman, Radcliffe-Brown, Hocart, Diamond Jenness, Gunnar Landtman y John W. Layard. En este contexto, Layard es el último de ellos, así como el último difusionista seguidor de Rivers.

Korsbaek, L. (2021). John Willoughby Layard (1891-1974). Antropólogo y psicoanalista haciendo etnografía en Melanesia antes de Malinowski. Iberoforum. Revista de Ciencias Sociales, Nueva Época, 1(2), 1-28, Artículos, e000190. 
Fue el secretario del Club Antropológico en Cambridge, del cual Rivers fue un frecuente conferencista; éste persuadió a Layard de quedarse en Cambridge un año más después de titularse y estudiar antropología.

Su primer y único contacto con el campo fue en 1914, cuando se preparó la 84. ${ }^{a}$ Asamblea de la Asociación Británica por el Avance de la Ciencia. El evento fue enorme; el número oficial de participantes fue 5044 (Withers, 2010, p. 47), pero no es posible decir cuántos antropólogos participaron, pues todavía no existía una sección de ciencias sociales y los antropólogos que participaron en especialidades como economía, estadística y pedagogía eran principalmente antropólogos físicos, arqueólogos, lingüistas y etnólogos. De todos modos, debe de haber sido casi toda la población de antropólogos en Inglaterra, lo que refleja la capacidad de gentes como $\mathrm{Ha}-$ ddon y Rivers para conseguir becas y viáticos para sus estudiantes.

Las sesiones se celebraron en las diferentes capitales de los estados que constituyen la federación de Australia, en aquel momento todavía parte del imperio británico. Layard viajó en el mismo barco que Rivers; en el transcurso del viaje, inició la Primera Guerra Mundial y Rivers decidió aplazar su regreso a Europa para dedicarse al trabajo de campo en Vanuatu, invitando a Layard a acompañarlo y participar en el trabajo de campo.

El principal resultado de la estancia de Layard en las islas fue la monumental monografía Stone Men of Malekula (Los hombres de piedra de Malekula), que es, al mismo tiempo, una inmensamente detallada descripción etnográfica de la vida en Vao y un análisis de un sacrificio ritual de jabalíes en la isla. El libro es un enredo de teorías difusionistas de Rivers y planteamientos psicoanalíticos de Gustav Jung, y es posible que plantee más preguntas que respuestas, pero antes que nada nos presenta un problema de tiempo.

La posición de Layard en la antropología social británica fue bastante peculiar: "fue el último de los difusionistas de una persuasión psicológica que basaban su marco teórico en Rivers. Hoy en día los únicos trabajos de Layard que se imprimen son los que escribió posteriormente como psicoanalista jungiano que, según me han contado, se encuentran en librerías ocultistas" (Gell, 1998, pp. 102-103).

En la antropología social británica fue marginal:

aunque John Layard estuvo entre los diecisiete presentes en la reunión fundacional, era ya en aquel entonces marginado en la antropología 
social, no obstante el hecho de que había hecho un año de trabajo de campo intensivo antes de que Malinowski llegara a las Islas Trobriandesas. Como alumno de Rivers y colega de Jung, Layard era al mismo tiempo antropólogo y psicólogo analítico. Como Rivers, pensaba que la antropología podría proporcionar materiales para la consideración de los procesos mentales, pero a diferencia de Rivers pensaba en esos procesos en términos de arquetipos, que los antropólogos "a pesar de estar rodeados por ellos en cada aspecto de su trabajo, de una manera tan curiosa los ha ignorado a través de una concentración en factores externos, a diferencia de los internos que siempre los acompañan a cada paso". (MacClancy, 1986, p. 50)

Hacia el final de su vida, Layard siempre explicaba su marginación, señalando que "no sabían de la psicología", y es muy posible que tuviera razón. John Layard fue un antropólogo marginal y un psicoanalista marginal, seguidor del psicoanálisis de Gustav Jung; en efecto, a pesar de pertenecer a buena familia, fue marginal en todo en la vida. Y en su marginalidad tuvo una vida auténticamente cosmopolita.

\section{John W. Layard, vida y milagros}

Nació en 1891 en el sur de Inglaterra y estudió en Bedard, una escuela de internado progresista; después de pasar un año viajando en Europa, estudiando francés y alemán, se inscribió en Cambridge en 1909, en donde estudió lenguas modernas en el King's College.

En Cambridge, paralelamente a sus estudios de lenguas modernas, se afilió a una asociación de estudiantes, la Sociedad Herética, que se reunía en los aposentos de C. K. Ogden para discutir problemas como "las bases filosóficas de la lengua"; posteriormente, fue el secretario del Club Antropológico, también bastante esotérico y especulativo, de la universidad. Layard se quedó el año en Cambridge, disfrutando su estancia, así que, cuando Haddon le consiguió una beca para ir a Australia, realmente fue con una preparación antropológica bastante básica, por no decir precaria.

Mientras Malinowski viajaba a Australia con Haddon, en el barco de vapor S. S. Orsova, rumbo a la 84. ${ }^{a}$ Asamblea de la Asociación Británica por el Avance de la Ciencia, John Layard viajaba con Rivers hacia la misma asamblea, pero en el barco S. S. Eurípides (una tarjeta postal con una foto del S. S. Eurípides y la firma de Rivers, Ernest Rutherford, Layard y otros 
científicos se vende por cinco mil dólares); las malas lenguas dicen que Haddon y Rivers fueron en barcos diferentes porque, a partir del discurso de Rivers en 1911 en Plymouth, en el cual abjuró del evolucionismo y se afilió al difusionismo, se había generado cierta distancia entre los dos teóricos. Layard se quejaba de que no fue posible hablar con Rivers, pues estaba corrigiendo el manuscrito de su The History of Melanesia (Rivers, 1914).

Rivers y Layard se instalaron en una pequeña isla, Atchin, en donde las actividades misioneras cristianas habían tenido muy poco efecto y los habitantes acababan de ahuyentar a un comerciante irlandés, así que el ambiente no era muy afable. Debido a la poca cordialidad de los salvajes, Rivers decidió abandonar la isla, dejando a Layard solo, mientras que él se dedicó a hacer trabajo más agradable desde el barco de los misioneros, La Cruz del Sur, y la villa del gobernador en otra isla:

Layard, que nunca supo si Rivers había arreglado ya de antemano la llegada de la lancha, se quedó así solo en Atchin, donde se quedó aproximadamente un año. Aparte de unas breves visitas a la isla vecina de Yao, donde disfrutaba la hospitalidad de un sacerdote católico, su único contacto con otros hombres blancos fueron las visitas cada dos meses de un vapor que daba servicio entre las islas. Así que muchos meses antes de que Malinowski levantara su carpa en las Islas Trobriandesas, Layard se encontró en una situación donde no tuvo otra salida que involucrarse profundamente en la sociedad de la isla Atchin. Estableciéndose con los nativos logró un sólido conocimiento de la lengua vernácula y se desempeñó durante un periodo de nueve meses como "novicio" honorario en las ceremonias de iniciación de los jóvenes y, en una ocasión bailó con los nativos, vestido solamente con un envoltorio de pene. (Langham, 1974, p. 238)

Layard era antropólogo, pero, al igual que Rivers, tenía una doble identidad científica: por un lado, como antropólogo y, por otro lado, como psicoanalista.

Aunque el joven Layard tenía una relación algo tirante con su padre, podemos suponer que tuvo una infancia feliz y armoniosa. Acerca de su estancia en la Universidad de Cambridge, después de que Rivers lo hubiera seducido para estudiar antropología en vez de continuar sus estudios de lenguas modernas, leemos que "fue uno de los periodos más felices en su 
vida" (MacClancy, 1986, p. 51); sobre el año que pasó en Atchin, escribe en su autobiografía: "Atchin fue mi paraíso -el único lugar donde realmente he estado feliz... viviendo con los nativos y disfrutando la vida con ellos" (Layard, s. f.).

Pero el regreso a la civilización occidental fue duro: Layard sufrió un choque cultural y tuvo necesidad de asistir a terapia. De una manera más general, la relación de Layard con el psicoanálisis fue interesante, pues lo llevó a ser primero paciente - o sea analizando- y luego analista activo.

Al principio se sometió a terapia con Rivers, quien acudió a los sueños como medio para resolver la crisis. El enfoque de Rivers había funcionado en el caso de Siegfried Sassoon, pero no así en el caso de Layard: "cuando Layard durante una de sus sesiones declaró que estaba enamorado de su mentor, Rivers pálido y casi temblando dejó la habitación y nunca volvió; evidentemente Rivers no se había dado cuenta del contenido homosexual de nuestra relación, probablemente de ambos lados" (Layard, s. f.).

Es posible que la terapia de Rivers haya acercado a Layard al psicoanálisis; al mismo tiempo, es posible que el trabajo con Layard le diera a Rivers ideas que posteriormente usaría en su trabajo de rehabilitación de soldados seriamente dañados en las trincheras durante la Primera Guerra Mundial.

Después, Layard tomó terapia con Homer Lane; luego, fue a Viena, en donde buscó al freudiano no ortodoxo Kremer y, en 1936, conoció personalmente a Carl Gustav Jung, quien había acudido a Londres para impartir una conferencia; este encuentro tuvo algunas consecuencias.

Podemos suponer que uno de los resultados de su estancia en Berlín fue su sostenida relación con el mundo psicoanalítico a través de Eranos, una organización de psicoanálisis, simbolismo y antropología fundada en 1933 en el sur de Suiza, en cuya revista -también llamada Eranos- publicó cinco artículos entre 1937 y 1958.

El análisis de Jung tiene una presencia muy limitada en la antropología británica, más en las tendencias especulativas del evolucionismo y del difusionismo, y menos en la antropología basada en trabajo de campo. Lo notable de la antropología psicológica jungiana de Layard es, tal vez, que se combina con un excelente nivel de trabajo de campo.

La relación de la antropología británica con el psicoanálisis siempre ha sido problemática, pues "la resistencia a la psicología se relacionó con la fuerte característica empirista que tanto marcaba la tradición británica en la antropología social" (MacClancy, 1986, p. 67). La primera generación 
de la etnografía en la antropología social británica, que fue dominada por médicos y naturistas, algunos de ellos con interés en y simpatía por el psicoanálisis -Haddon, los Seligman, Rivers y Jones, el introductor y biógrafo de Freud-, que desembocó en el enfoque de Malinowski, le dio algo de espacio a los diversos tipos de psicología. Sin embargo, a partir de 1937, la antropología británica sería una antropología estrictamente social, dominada por Radcliffe-Brown y sociológicamente orientada hacia Durkheim; como señala Adam Kuper (1989), no hubo ningún espacio para ningún tipo de psicología. John Layard estaba marginado y el psicoanálisis también.

John Layard pasó luego tres años en Berlín. Parece que ya tenía amistades por su primera visita a Europa como jovencito; un periodo permeado por la memoria de su terapia con Homer Lane en Inglaterra, su vida con un círculo de bohemios artistas y literatos homosexuales, así como su intento de suicidio en 1929 -hecho que se volvió un asunto literario, con variadas interpretaciones, pero siempre subrayando el resultado final: que John Layard sobrevivió; de allí el comentario de Evans-Pritchard: "Por supuesto, John Layard está loco, se metió una bala en la cabeza y no hizo diferencia alguna. Debe estar loco" (MacClancy, 1986, p. 65)-. De regreso a Londres, lo trató H. G. Baynes, quien había introducido la terapia de Carl Gustav Jung en Inglaterra. En una ocasión que Jung llegó a Londres para dar una conferencia acerca de "lo inconsciente colectivo", Layard se le acercó y se convirtió en su paciente.

\section{John W. Layard, su trabajo de campo}

La etnografía de Layard se inscribe en una larga y sólida tradición de trabajo de campo y parentescología en una parte del lejano oriente. ${ }^{3}$ El evolucionismo se había movido en el mundo entero y había tenido como su principal tema el nacimiento y el desarrollo de las religiones; los sistemas de parentesco habían ejercido muy poca atracción sobre los evolucionistas - tal como lo decía Edward Said, a los orientalistas no les interesaban los orientales más que como espejo de los occidentales-. Con la defunción del evolucionismo "clásico" y el surgimiento de los estudios sincrónicos de los sistemas de parentesco, esos estudios se concentraron en tres regiones: Australia, la Nueva Guinea y Melanesia.

3 Viví un tiempo con un australiano; él me explicó que, por razones obvias, lo conocía como "el norte cercano".

Korsbaek, L. (2021). John Willoughby Layard (1891-1974). Antropólogo y psicoanalista haciendo etnografía en Melanesia antes de Malinowski. Iberoforum. Revista de Ciencias Sociales, Nueva Época, 1(2), 1-28, Artículos, e000190. 
La etnografía de John Layard coincidió con el nacimiento del interés por la etnografía de los sistemas de parentesco (parcialmente iniciado por Rivers) y se desarrolló exclusivamente en Melanesia, específicamente en una parte muy circunscrita de Melanesia: la isla de Malekula y las pequeñas islas vecinas. Puede ser que la parte más trascendental de la etnografía (y antropología) de Layard será su parentescología.

Cuando Haddon llegó a Melanesia en 1914 (acompañado por Malinowski), tenía, originalmente, el proyecto de llevar a cabo un sondeo general de la costa de Nueva Guinea; la armada británica le había prometido el apoyo de una lancha artillada pero, como la Primera Guerra Mundial comenzó exactamente cuando los alrededor de 300 científicos sociales británicos iban llegando a Australia y Melanesia, la armada tenía otras cosas en qué utilizar sus lanchas artilladas y Haddon tuvo que abandonar su proyecto, en el cual había incluido a Layard como ayudante. En lugar de este proyecto de sondeo, le sugirió a Layard que podía acompañar a Rivers en su trabajo de campo.

Layard se quedó en Atchin un año, hasta octubre de 1915. Su autobiografía, Study of a Failure (Estudio de un fracaso), es diferente de la de Malinowski (por ejemplo, no ha sido publicada): "no hay nada de ambivalencia como la encontramos en la autobiografía de Malinowski, nada de la agresividad, nada de extrañar regresar a la humanidad blanca" (MacClancy, 1986, p. 52).

El trabajo de campo más importante de Layard -y el único en toda su vida - fue esta investigación de un año (1914-1915) que Rivers lo sedujo a llevar a cabo en la isla Atchin y las islitas vecinas; su principal publicación sería el famoso Stone Men of Malekula (Los hombres de piedra de Malekula), que se basa en aquel trabajo de campo, aunque no fue publicado hasta 1942.

La etnografía de Layard cubre tres, tal vez cuatro, localidades en Malekula: su principal trabajo de campo lo hizo en la isla Atchin, pero, ya que los habitantes de ésta tenían huertas en Malekula, al otro lado del estrecho, hizo también algo de trabajo de campo en "tierra firme"; hizo una pequeña expedición de unas cinco semanas a la isla Vao, en donde el misionero católico le dio hospedaje y de donde viene su mamotreto Stone Men of Malekula. Es realmente fantástico que haya logrado hacer una monografía de más de 800 páginas con base en cinco semanas de trabajo de campo. El último lugar en donde hizo trabajo de campo fue en el South West Bay (Bahía del suroeste), del que produjo un rico texto etnográfico con base en sólo una 
semana de trabajo de campo. La generosidad de Layard quedó de manifiesta cuando se dirigió al joven estudiante Deacon y le regaló sus apuntes de South West Bay; cuando falleció Deacon, a la edad de veinticuatro años, y se publicaron sus notas de campo, a cargo de Camila Wedgewood, surgió un pleito de lo más común entre antropólogos acerca del derecho a las observaciones; a esto volveremos más adelante.

\section{Imagen 1. Mapa de Malekula}

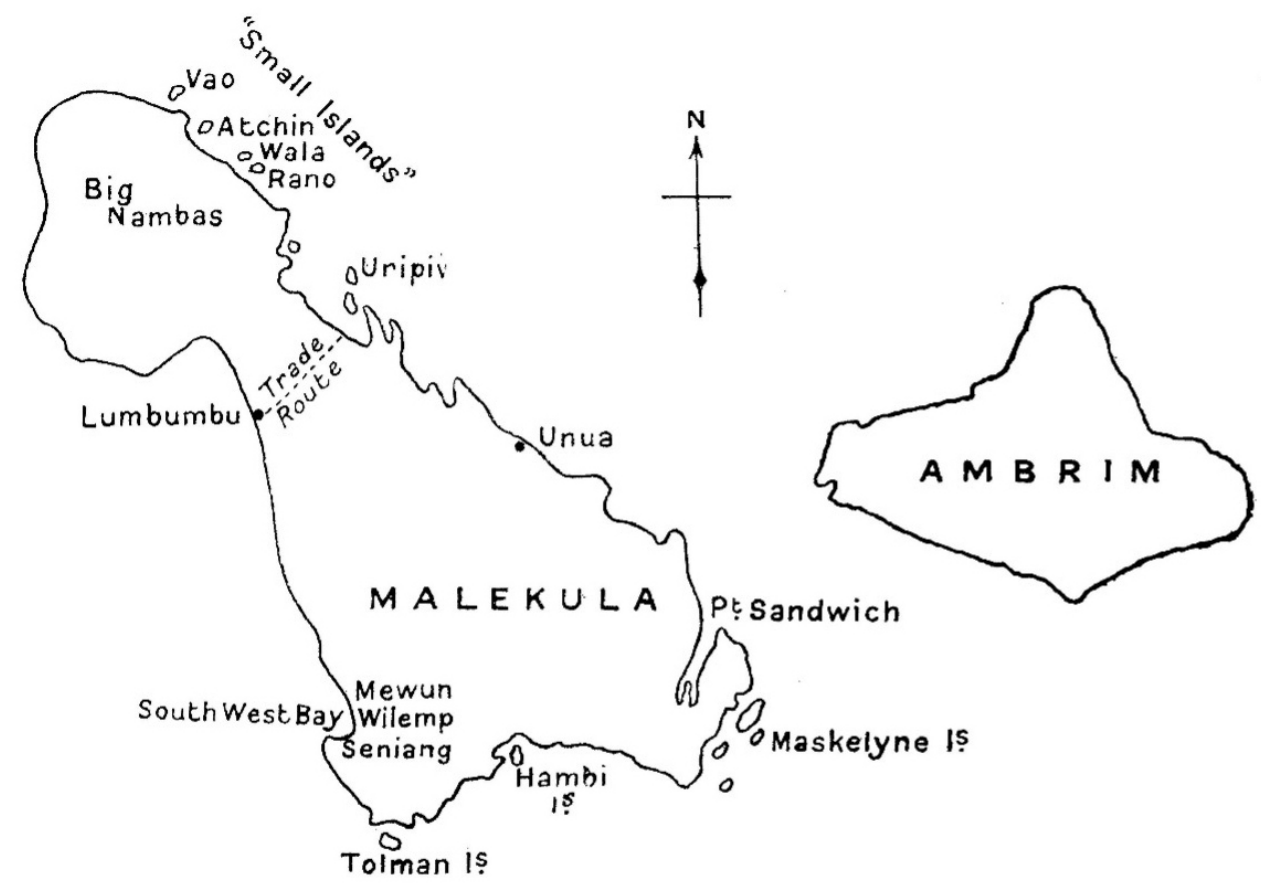

Fuente: Layard, 1928, p. 141

\section{Atchin}

De Atchin, el centro de las actividades de Layard, existe una muy interesante etnografía con el título "Atchin hace veinte años" (Layard, 1936a). La etnografía tiene dos partes: la primera es una descripción etnográfica atemporal, basada en las observaciones de Layard en 1914, mientras que la segunda es una especie de contemplación histórica acerca de la llegada del hombre blanco y basada en los conocimientos de Layard en 1936. 
La etnografía de Layard de la isla de Atchin empieza, como suele suceder, con una descripción geográfica de la isla: es una de seis muy pequeñas islas -las demás son Vao, Wala, Rano, Uripiv, Uka ${ }^{4}$ - que se encuentran frente a la costa noreste de Malekula, que es una isla de unos 120 kilómetros de largo y 50 kilómetros de ancho; las pequeñas islas están separadas de Malekula por un estrecho que mide alrededor de un kilómetro. Cada isla mide aproximadamente un kilómetro cuadrado y tiene una población de 400 a 500 personas. Sus habitantes se consideran a sí mismos como "gente del mar", a diferencia de la "gente de la selva" ("bush folk") de la isla Malekula. Las islas "forman un grupo étnico", pero no cuentan con un gobierno central y están divididas en aldeas que tienen, cada una, su propia lengua.

Hay tres rasgos en la etnografía y la realidad de este pueblo que llaman la atención. El primero es que la población se encuentra dividida a la mitad en sus aldeas en cada isla. Podemos ver esta investigación como una inspiración de los entonces recientes estudios de parentesco en Australia (Fison y Howitt, 1880; Spencer y Gillen, 1899, 1904).

El segundo es un ritual llamado "maki", que está estrechamente relacionado con la organización social:

el maki completo que, como ya hemos señalado, está alternantemente ejecutado por hombres de generaciones alternantes, es un rito compuesto prolongado que dura, en su forma extendida, de cualquier lugar entre quince y treinta años, durante los cuales se lleva a cabo una serie interminable de ritos menores, cada uno involucrando el sacrificio de numerosos jabalíes colmilludos e implica un enorme gasto de alimentos para las fiestas. (Layard, 1936a, p. 345)

\section{El tercer rasgo es que}

esta compleja organización social y habilidad lingüística presupone una notable inteligencia promedio y está acompañada por un sentido genealógico e histórico que no tiene su igual en Melanesia, se puede comparar, en lo que al Pacífico se refiere, con el de los polinesios, con quienes este pueblo tiene íntimas relaciones culturales. En Atchin,

4 Las islas son conocidas colectivamente como "Las pequeñas islas", de la sexta isla, Uka, no se tiene información etnográfica. 
habían genealogías exactas, que se pueden checar por varios medios, y que alcanzan hacia atrás diecisiete generaciones e incluyen relatos detallados de las hazañas de muchas de las figuras que aparecen en ellos así como de los antepasados de varias familias extensas interrelacionadas que entre ellas constituyen la organización de la aldea. (Layard, 1936a, p. 343)

Los efectos de la llegada del hombre blanco son muchos; el tono del relato de Layard es profundamente melancólico. En el momento de sus observaciones, en 1914, la situación en la isla era adversa, con una reducción del número de habitantes; Layard vio dos causas de ello: la introducción del rifle (mosqueta) y las enfermedades causadas por la llegada del hombre blanco. Haciendo referencia a la teoría de Rivers (1922) sobre el efecto del pesimismo como parte del proceso de colonialismo, Layard (1936a) señala que "puedo decir de manera definitiva que en aquel momento una creencia general entre los ancianos y algunos de los jóvenes era que la raza estaba condenada" (p. 350) y que algunos de los jóvenes decían "si la situación es así, ¿para qué sirve criar niños?" (p. 351). Sin embargo, con la ventaja de la retrospección, observa, en 1936, que algunos de los jóvenes que nunca habían conocido los tiempos anteriores a la llegada del hombre blanco tenían una visión templada de las bendiciones de éste: "ellos eran los hombres que estaban decididos a rehabilitar la cultura de Atchin, y al mismo tiempo integraron a la cultura de Atchin tales nuevos elementos que aparentemente no estaban demasiado en conflicto con la cultura nativa" (Layard, 1936a, p. 351).

Haciendo referencia a Lawak, su principal amigo e informante, "el hombre más influyente en la isla", Layard señala:

tengo que confesar que en 1914, aunque admiraba sus esfuerzos, tenía poca esperanza de su último éxito. El tiempo ha mostrado que no fue así. La población se ha incrementado y se sigue incrementando. Es gracias a hombres como Lawak, quienes, a través de su conocimiento del hombre blanco y sabiendo cómo tratar con él, que tenían esperanza en el futuro pero al mismo tiempo preservaron su independencia de mente y amor a su propia cultura, que hoy contamos con un incremento de la población y un renacimiento de la cultura. (Layard, 1936a, p. 351) 
Otra expresión del optimismo de Layard es que "el autor espera publicar un relato detallado de la organización social, lengua, música y actividades marítimas de los habitantes de las islas frente a la costa noreste de Malekula, en una serie de volúmenes con el título de Stone Men of Malekula. El primer tomo, dedicado a la isla de Vao, debe aparecer este año en el otoño" (Layard, 1936a, p. 351). De la serie se publicó sólo ese primer tomo dedicado a la isla de Vao, y solamente unos años más tarde (Layard, 1942).

\section{Vao}

La etnografía de la isla Vao es paradójica, pues el centro de trabajo de Layard fue la isla Atchin, en donde hizo el trabajo de campo más extenso y más sólido; sin embargo, la etnografía más acabada, su monografía de mas de 800 páginas, Stone Men of Malekula (1942), es de la vecina isla Vao. Por cierto, las primeras palabras de su monografía son "este volumen es el primero de una serie que presentarán los resultados del trabajo que se ha llevado a cabo en los años 1914 y 1915 en las pequeñas islas frente a la costa noreste de Malekula en las New Hebrides", hoy Vanuatu (Layard, 1942, p. XVII). Sin embargo, el destino y otras fuerzas no le permitieron acabar y publicar su obra, a lo que volveré en las conclusiones.

En el prefacio al libro, Layard (1942) señala: "pronto se nos hizo claro que la cultura de esas islas era cualquier cosa menos sencilla", y habla acerca de "los dos principales elementos culturales en esas islas, en la materia de parentesco, donde se estaban obrando cambios sustanciales, que llevaron a guerras frecuentes debido a nuevas influencias que afectaban la regulación matrimonial y, antes que nada el ritual megalítico del cual nuevas formas constantemente se adquirieron desde el sur" (pp. XVII-XVIII).

Layard explica de qué manera su atención se dirigió hacia el ritual conocido como "maki" en la isla: primero por los intereses de Rivers por construcciones megalíticas y luego porque

con nueva evidencia se acababa de descubrir culturas megalíticas contemporáneas a través de la publicación del Dr. Speiser de los resultados de una expedición al norte de las New Hebrides, donde mencionó la existencia de un gran número de construcciones megalíticas, tales como dólmenes y monolíticas, que todavía fueron erigidos en el curso de sus ritos. (Layard, 1942, p. XVII) 
Más tarde, este interés fue confirmado y reforzado por sus conversaciones con varios misioneros católicos, tanto en Vao como en Francia.

La parentescología de John Layard en Stone Men of Malekula, reflejo de una especialidad antropológica introducida por Rivers, viene a ser, al mismo tiempo y de una manera curiosa, la motivación e inspiración de los estudios de uno de los jóvenes genios de la antropología británica, y la continuación de la etnografía del mismo Bernard Deacon. El parentesco ocupa dos capítulos del libro, de los cuales el primero es un resumen de los conocimientos disponibles al momento del estudio - podemos discutir si ese momento fue el año 1914 o el año 1942, pero las principales referencias son los estudios de Spencer y Gillen $(1899,1904)-$.

La parentescología está presente en la monografía de Vao, pero contiene mucho más - cultura, demografía, organización social-; sin embargo, el tema principal es el ritual "maki", que se encuentra en toda Vanuatu, aunque con variaciones.

El ritual maki - presente en las culturas costeras de Malekula, es decir, principalmente en las pequeñas islas, aunque también en las comunidades que persisten más al sur por la costa oriental de la isla y, como se verá, en el South West Bay- abarca, en términos generales, las páginas 203-684 de la monografía, aunque en otras páginas se agregan descripciones de aspectos más particulares del ritual. Podemos comprimir una descripción general como sigue:

El maki es un tiro propiciatorio en el cual, a través del sacrificio de jabalíes colmilludos el sacrificador adquirió el ta-mat (alma) del jabalí, evitando así la aniquilación por el espíritu guardián después de la muerte, y al mismo tiempo exalta los espíritus ancestrales y se asegura un lugar a su lado en lo más allá, subiendo paulatinamente en los grados después de cada sacrificio exitoso. Cada sacrificio representa un renacimiento manifestado en un nuevo nombre y un nuevo rango, y fue seguido por un periodo de seclusión. (Layard, 1942, p. 270)

El rito dura en su totalidad entre quince y veinte años, así que sería justo llamar a Layard "el antropólogo del ritual", tal como he señalado acerca de Hocart en otra ocasión (Korsbaek, 2019). 


\section{South West Bay}

Vale la pena citar un fragmento de la introducción a un artículo temprano de John Layard, de 1928, cuando ya había llegado a Londres la noticia de la muerte del muy joven antropólogo A. Bernard Deacon, pues nos da información acerca de la seriedad del trabajo de Layard y nos da unas pistas para el desarrollo de la antropología británica en Melanesia:

Gran parte del tiempo del Sr. Deacon lo pasó en South West Bay, Malekula, y es en la esperanza de la inminente publicación de sus apuntes que el Dr. Haddon me pidió que contribuyera con los resultados de una breve visita que hice a aquel distrito en mayo de 1915. La base de mi propio trabajo de campo intensivo fue en Atchin, una pequeña isla frente a la costa noreste, y fue en el marco de un sondeo general de Malekula que se me permitió hacer trabajo de campo de una semana en el South West Bay [...] Es evidente que mis apuntes son el resultado del tiempo extremadamente corto a mi disposición, y titubearía en publicarlos si no fuera por la honesta solicitud del Sr. Haddon. (Layard, 1928, pp. 140-145)

Una caracterización general del South West Bay la encontramos en otra publicación de Layard:

Existía, sin embargo, otro centro megalítico, en apariencia más antiguo que mantenía una comprensión más profunda del significado del interior de la vida y la muerte... esta era la montañosa zona costera que limitaba ambos lados de la entrada larga y arenosa llamada la Bahía del Suroeste. Aquí la vida ritual megalítica era anteriormente mucho menos comunal y más un evento individual, en el que la ascensión progresiva en la jerarquía religiosa a la que pertenece cada varón. En Malekula representaba una "escalera espiritual" mucho más clara para el aspirante individual. (Layard, 1928, pp. 140-145)

La cosecha etnográfica de su extremadamente corta estancia en South West Bay, una semana, es impresionante y cumple la misma función que el artículo de Atchin: sustituir un volumen entero que nunca se publicó.

La etnografía general es somera, más bien mínima; primero, caracteriza a la población costera general: 
muestran una gran diversidad cultural, pero comparten ciertos rasgos destacados, entre los cuales se encuentran: gobierno por medio de la opinión pública centrada en los ancianos de la aldea; los hombres visten un cubre-pene tejido y las mujeres visten una faldita apretada alrededor de los muslos; incisiones; tambores con hendiduras; organización alrededor de una casa club, y la institución de otorgamiento público menggi, mangki o maki (en el South West Bay menggi). (Layard, 1928, p. 141)

Después, procede a presentar, también casi taquigráficamente,

los rasgos especiales que distinguen la cultura del South West Bay de las culturas del norte de Malekula:

I. Deformación de cabeza y nariz

II. Modelando el cráneo de los muertos distinguidos de arcilla, con el fin de representar los rasgos del difunto con la mayor exactitud posible, montándolos en cuerpos artificiales y guardando estas efigies en la casa club

III. El uso, para fines de magia, de cerámicas de una hechura que los indígenas desde mucho tiempo ignoran y que atribuyen a

IV. Una raza de piel blanca llamada Ambat que consideran como sus antepasados. (Layard, 1928, p. 142)

Acerca del "menggi" en South West Bay escribe que:

consiste en una serie de grados a través de los cuales un hombre avanza en rango, y la membrecía abarca toda la población. En todos los casos el novicio debe ser introducido en su nuevo rango por alguien que ya lo ha adquirido, a quien le hace el pago apropiado. El punto central de cada ceremonia es la erección de una imagen de madera, un monolito, un dolmen o una plataforma de piedra, o una combinación de esos elementos. En el cual el novicio sacrifica un jabalí propiedad del introductor. Luego adopta el novicio un nuevo nombre y un nuevo fuego y recibe las insignias de su nuevo rango. (Layard, 1928, p. 142)

5 Es imposible resistir la tentación a llamar la atención sobre la similitud de esta institución con la institución conocida como el sistema de cargos en Mesoamérica: ambas son sistemas 
Layard subraya la similitud de esta ceremonia con la correspondiente ceremonia en las Islas Banks, sólo que allá se lleva a cabo en profunda secrecía, mientras que en Malekula es totalmente pública: "de hecho, las piedras y las imágenes relacionadas con el menggi constituyen el rasgo más destacado que capta la vista al entrar en una aldea en Malekula" (Layard, 1928, p. 142).

"Aunque las características fundamentales de la institución son esencialmente las mismas en todas partes de la costa, permite una infinitud de variaciones, no solamente en detalles sino también en el diseño general de la ceremonia" (Layard, 1928, p. 142). Las variaciones se encuentran en el aspecto cuantitativo de la institución y tiene que ver con el número de rangos:

en Unua en el centro de la costa oriental de Malekula hay alrededor de cinco y solamente pocas gentes alcanzan el grado de "mal", y a un costo muy alto. Y hacia el norte y el sur de este punto hay una notable divergencia en el desarrollo de la institución en sentidos diametralmente opuestos: en el norte se reduce el número de rangos, mientras que en el sur hay una tendencia a aumentar el número.

Por un lado, un hombre de Unua podría sufrir un fuerte choque cuando, al moverse hacia el norte por la costa, se da cuenta de que cualquier fulanito, que tenga la necesaria edad para haber participado en la ceremonia en absoluto, es el orgullo poseedor de este título. Aquí el número de grados ha sido reducido a uno solo, con el título supernumerario de "meldek" agregado más arriba.

Por otro lado, en el distrito de Sulol, en Ambrim, el número ha crecido hasta diecisiete, y en South West Bay es hasta más alto. Cada uno de esos desarrollos parecen deberse a la misma causa, o sea la pasión de los nativos por la multiplicación de ceremonias. En el primer caso, esa se manifiesta en el número de grados. En el segundo ha asumido la forma de una elaboración del grado central, de "mal", con la consecuente eliminación de los demás. (Layard, 1928, p. 143)

de roles que abarcan la totalidad de la población en la comunidad y confieren dignidad y rango a sus participantes (véase, por ejemplo, Korsbaek, 1996). Sin embargo, no tengo la más mínima intención de postular la existencia de una difusión o migración entre las dos áreas culturales.

Korsbaek, L. (2021). John Willoughby Layard (1891-1974). Antropólogo y psicoanalista haciendo etnografía en Melanesia antes de Malinowski. Iberoforum. Revista de Ciencias Sociales, Nueva Época, 1(2), 1-28, Artículos, e000190. 
Su muy rica etnografía de South West Bay termina con la nota de que "toda la historia del cambio en Atchin será publicada en mi libro acerca de esta isla" (Layard, 1928, p. 143); sabemos ahora que el libro nunca fue publicado y tal vez nunca fue escrito en una forma acabada.

\section{La etnografía de Layard}

En la etnografía de Layard, se reflejan los principales intereses de su día, en una agenda muy similar a la de su casi contemporáneo Maurice A. Hocart: mucho de la magia y de la religión, pero también mucha parentescología, música y danza; todo mirado a través del filtro del difusionismo introducido por Rivers en 1911.

El canto y la danza ocupan un lugar importante en la etnografía de Layard, tal como se manifiesta en un breve texto de 1944: "como en otras partes de Melanesia, el genio artístico de los nativos se puede expresar en una variedad de diferentes maneras [...] en el ritual esotérico de sociedades secretas, tal como en las pequeñas islas y distritos costeros de Malekula se expresa principalmente en el drama, la música y en la danza formal" (Layard, 1944a, p. 121).

Dedica mucha atención a los instrumentos musicales, sobre todo al tambor; un tipo particular de éste, el tambor con hendidura (en inglés, slit drum), juega un papel importante en la vida ritual en la isla: "los tambores y los monumentos de piedra ambos representan a los ancestros, cuyas 'voces' hablan a través de los tambores como parte del proceso de producción del alma" (Layard y Haidy, 2005, p. 195).

Algunas otras características de su etnografía llaman la atención. En primer lugar, muestra un enorme respeto por la cultura de Malekula; en el mismo texto corto antes mencionado, nos da una imagen de ésta -equiparándola con la cultura occidental- como una cultura con todo, hasta música sinfónica.

Como un reflejo de su respeto, se nota también que su etnografía ha llegado a ser una parte de la cultura actual de Malekula, pues la monografía Stone Men of Malekula

circulaba localmente, en su totalidad o parcialmente, en fotocopias, con frecuencia secretamente. Ha sido traducida en parte a la lengua franca bislama y al francés (los habitantes de Vao son francófonos).

El dramático volumen está en el centro de muchas disputas contem- 
poráneas acerca de los derechos de propiedad o liderazgo y se ha convertido en el foco de un kastom polici (política de costumbre) del consejo de jefes en Vao. (Geismar, 2009, pp. 199-200)

Esto evidentemente plantea la espinosa pregunta: ¿cuál es el papel de un antropólogo imperial que se solidariza con el pueblo colonizado?, ya que las islas en 1980 se convirtieron en la república Vanuatu.

La sombra de este difusionismo la vemos en su magnum opus, Stone Men of Malekula. Los vicios del difusionismo los saca a relucir Ashley Montagu en una reseña en 1944; antes de alabar las virtudes etnográficas del libro, destaca algunos comentarios de Layard como que el miedo de los nativos a serpientes perfectamente inocentes "se debe posiblemente a una memoria racial de origen continental, donde las serpientes son físicamente peligrosas" (Montagu, 1944, p. 43) o cuando habla de los que critican a Perry y Elliot Smith y su difusionismo como detractores, pero "Sr. Layard por suerte pronto se dedica a la descripción de los hechos y se olvida de sus prejuicios" (Montagu, 1944, p. 43).

Una evaluación general, no muy simpática del libro de Layard, nos cuenta que

aunque Stone Men of Malekula sí contiene una buena cantidad de interesante material de parentescología, incluyendo algunos métodos ingenuos diagramáticos para la representación de sistemas de clases de matrimonio, la fecha tardía de su publicación, su limitada circulación debida a la Segunda Guerra Mundial, y su propósito de presentar la difusión cultural, ha reducido a un mínimo su influencia. (Langham, 1981, p. 205)

\section{Las publicaciones de John W. Layard}

John Willoughby Layard - quien nació en el sur de Inglaterra en 1891, hizo trabajo de campo en Melanesia de 1914 a 1915, intentó suicidarse en Berlín en los años 1930 y finalmente falleció en Oxford en 1974- escribió en 1936:

el autor espera publicar una relación detallada de la organización, la religión, la lengua, la música y las actividades marítimas de los habitantes de las pequeñas islas fuera de la costa noreste de Malekula, en 
una serie de volúmenes intitulados Los hombres de piedra de Malekula.

El primer volumen, tratando la isla de Vao, está por aparecer este otoño.

(Layard, 1936a, p. 351)

Prestando atención a algunos detalles y a algunos de los imponderables de la vida humana, como decía Malinowski, estas palabras sirven como una adecuada introducción a una breve discusión de las amplias publicaciones y los obstáculos que se le presentaron a John W. Layard en el transcurso de su vida cosmopolita y atormentada.

Después de su único trabajo de campo, en Vanuatu en 1914 y 1915, tardó bastante en publicar sus observaciones pues, a su regreso a una Inglaterra en guerra, su vida no fue más que sufrimientos y decepciones por un buen rato. Entre otros eventos, intentó enlistarse y participar en la guerra, pero fue rechazado, primero en Australia y después en Inglaterra; además, se encontraba en terapia constantemente, tal como se señaló antes. A pesar de esas adversidades, después de 1928 tuvo una producción de publicaciones sorprendentemente sostenida. En las siguientes páginas, me limitaré a sus publicaciones antropológicas, de las cuales la mayor parte se relaciona con Malekula.

Su primer artículo es de 1928 y tiene que ver con las intrigas en el escenario de la antropología británica, pues empieza así: "La etnología ha sufrido una sensible pérdida con la trágica muerte del Sr. Bernard Deacon, en la víspera de su salida de las New Hebrides después de un largo periodo de trabajo de campo" (Layard, 1928, p. 140). Bernard Deacon, quien también hizo trabajo de campo en la isla Malekula, falleció de alguna fiebre tropical (blackwater feber) en 1927 a la edad de veinticuatro años; Layard era un obvio candidato para la tarea de editar sus notas de campo; sin embargo, Haddon y Radcliffe-Brown le asignaron el trabajo a Camilla Wedgwood, a pesar de que Layard se ofreció para ello. La razón habría sido que Haddon y Radcliffe-Brown temían que Layard "estuviera en complicidad con Elliot Smith, pues éste ya le había encargado a John Layard el trabajo de darle forma al material de Rivers de Ambrym" (Langham, 1981, p. 237). Elliot Smith era el jefe de los enemigos, los difusionistas, con los cuales Rivers se había aliado en 1911. El trabajo terminó en un pequeño escándalo, pues Wedgwood había tratado el material con descuido y cometió el error de atribuir algunos materiales de Layard a Deacon. En la edición, Haddon tuvo que dejarle a Layard señalar algunas de las imprecisiones en un "prólogo 
complementario" (Langham, 1981, p. 242). De este evento surgió, entonces, el artículo "Degree-taking rites in South West Bay, Malekula" (Layard, 1928), que es netamente la etnografía general y la descripción del rito que le interesaba a Layard en la Bahía del Suroeste de Malekula.

En 1930, Layard publicó un texto acerca de la etnografía de los brujos llamados "bwili" bajo el título de "Malekula: Flying tricksters, gods, ghosts and epileptics"; un artículo que nos muestra a Layard como etnógrafo, revelando tanto su fuerza como sus debilidades en la etnografía. En lo temático se acerca mucho a A. M. Hocart, quien trabajó en la misma región unos años antes (cuando Layard trabajaba en Malekula, Hocart estaba en las Islas Fiji; deben de haber regresado a Inglaterra más o menos al mismo tiempo: Layard para ser rechazado como voluntario, Hocart para llegar al rango de capitán en el ejército británico).

Entre 1937 y 1956, Layard publicó una serie de artículos en la revista Eranos Jahrbuch (Anuario Eranos) - podemos suponer que, por intervención de Carl Gustav Jung-, de los cuales algunos fueron escritos en alemán. También podemos suponer que son consecuencia de su encuentro con Jung en Londres en 1936 pues, como era de esperarse, tomando en cuenta las características de la revista Eranos, todos son interpretaciones de su material etnográfico de Malekula en clave del psicoanálisis de Jung. Por otro lado, podemos ver la secuencia de publicaciones en la Eranos como reflejo de su proceso de alejamiento de la antropología después de la publicación de su obra magna Stone Men of Malekula, pues la falta de atención al libro debe haber sido una enorme decepción para Layard.

El primero de los artículos está en alemán y tiene que ver con la vida después de la muerte (Layard, 1937a); como otros de sus textos, de nuevo es testimonio de su cercanía temática a Hocart. El segundo de esos artículos es de un tema clásico en la antropología, el tabú de incesto, y un tema del psicoanálisis de Jung, el arquetipo de la virgen (Layard, 1945). El penúltimo de sus artículos en Eranos es el único que existe en español; versa acerca de la construcción del hombre en Malekula (Layard, 2004/1948). El último de los textos de Eranos es también una combinación de un tema clásico de la antropología británica, el sacrificio, y el problema de transferencia en el psicoanálisis (Layard, 1956).

La revista Eranos Jahrbuch, que nació en 1933 (y sigue existiendo), fue el anuario del proyecto de investigación y discusión Eranos (en griego, significa la comensalidad), fundado por Rudolf Otto en Locarno en Suiza; 
dicho proyecto pronto se convirtió en un centro de estudios del psicoanálisis según las teorías de Carl Gustav Jung, así que no es disparatado considerarlo como el centro de estudio y difusión del psicoanálisis jungiano.

Unos años más tarde, Layard publicó un artículo retrospectivo con el título "Atchin twenty years ago" (1936a), que es realmente una versión comprimida del tomo de Atchin, si nos imaginamos que Layard hubiera publicado sus cuatro volúmenes de etnografía de las pequeñas islas, tal como lo había planeado.

En los mismos años 1935-1936 se dedicó a un nuevo interés: el estudio del laberinto, que vio como un símbolo de extensión universal. De este tema publicó tres artículos, en estrecha relación con su visión difusionista (Layard, 1935, 1936b, 1937b). Tal vez los podemos ver como ensayos de su obra principal, The Stone Men of Malekula, de cuya edición final se ocupaba en el mismo periodo.

Finalmente, se organizó para editar y publicar su magnum opus, Stone Men of Malekula, que sería publicado hasta 1942. Es impresionante en todo, aun en cuanto a dimensiones: a pesar de las restricciones de la guerra, tiene más de 800 páginas (mi versión en pdf es de 902 páginas) y es un digno monumento a su antropología, aun siendo inacabado.

También en plena guerra mundial, publicó dos notables artículos: el primero, "Song and dance in Malekula" (Layard, 1944a), nos da, en sólo una página una impresión de la amplitud de la cultura autóctona de Malekula que, como ya se mencionó, contiene hasta música sinfónica; el otro es un artículo acerca del sistema de parentesco en Malekula interpretado en clave del psicoanálisis de Jung, haciendo referencia a la estructura psicológica del hombre moderno (Layard, 1944b); hay que ver este último artículo en conjunto con un manuscrito de 800 páginas que nunca publicó acerca del sistema de parentesco en Vao.

Aun en los años cincuenta, es como si Layard todavía se encontrara en Malekula: publicó un artículo acerca de ritos de iniciación sexual, "The Pilgrimage to Oba. An Atchin sex-initiation rite" (Layard, 1951), un tema que le ocupó toda la vida después de su trabajo de campo.

Todo el decenio de 1950 se dedicó a estudiar de nuevo el papel de los jabalíes en la vida social y ritual de Malekula, otorgándole el mismo lugar al jabalí en Melanesia que ocupa el ganado en la vida social y ritual en las sociedades bantú, en el este de África. Al final de esta década, publicó un artículo que se puede ver como una alusión existencial a un problema que 
lo siguió toda la vida, para bien y para mal: su propia homosexualidad (Layard, 1959).

El resto de su vida, hasta el año 1974, se dedicó principalmente al psicoanálisis sin otra referencia a Malekula que lo sobreentendido -en cierto sentido, Malekula estaba presente en toda su vida-; la única obra de este periodo que tiene importancia para esta semblanza de su vida antropológica son sus memorias, que no publicó y que llevan el título significativo Study of a Failure (Estudio de un fracaso) (Layard, s. f.).

De la generación de antropólogos cuyos miembros iniciaron el trabajo de campo en la antropología británica entre 1898 y 1922, John Layard fue el más longevo pues, a excepción de Diamond Jenness, quien falleció en 1969, la mayoría falleció alrededor de 1940; Layard murió en 1974, un hecho que produjo la curiosa situación del trabajo de campo de Bernard Deacon en Malekula y la publicación de su material de aquella isla. En cierto sentido, la publicación, con las complicaciones mencionadas, fue un éxito, a tal grado que se hizo una segunda edición en 1970. Al igual que la primera edición de 1934, esta segunda edición ha sido severamente criticada:

la edición original de este clásico monumental ha sido ligeramente modificada en su formato [...] han sido colocados asteriscos en el margen del texto para identificar aquellas partes a las cuales los comentarios de Layard hacían referencia [...] el libro sigue siendo una colección de notas del campo de Layard, notas del campo de Deacon y las interpretaciones editoriales de Wedgwood a ambas; el problema es que ya no es posible en la mayor parte del libro decidir qué es qué. (Dunn, 1974, pp. 910-911)

En una carta a Haddon, Deacon (1934) comentó acerca de Malekula: "pienso que le golpea a uno la extraordinaria complejidad de la diferenciación cultural [...] es sumamente notable el aislamiento de los grupos" (p. XXI-XXII). Esta opinión se encuentra en agudo contraste a otra incluida en el texto editado:

este sondeo cursorio refiere principalmente a la gente en el South West Bay, acerca de los cuales tenemos mejores conocimientos que de los demás distritos, pero se puede tomar como típico de todas partes de la isla [...] en combinación con los primeros y últimos capítulos del li- 
bro, muy al estilo de Rivers, constituyen evidencia de una inaceptable premisa difusionista, compartida por Layard, Deacon y Wedgwood, y posiblemente aplicada de la manera más crítica por Wedgwood [...] hasta distorsiona gran parte del material del libro. (Dunn, 1974, p. 911)

El último detalle que vale la pena mencionar en relación con sus publicaciones es una breve noticia en la revista Pacific Arts: "Documentos de Layard donados a Melanesian Archive por su hijo Richard Layard, profesor de economía laboral de London School of Economics", en la que se apunta que "los Layard papers son de mayor interés para estudiosos de Melanesia, pues más allá de las notas de campo, fotos y otros materiales de la expedición, contienen un manuscrito a máquina de más que 800 páginas acerca del sistema de parentesco, y la organización social de Atchin, junto con otros proyectos de libro parcialmente acabados" ("PIC Newsletter", Pacific Information Centre, University of the South Pacific, 1990, p. 106); la nota termina con el comentario de que "se puede obtener más información con el Profesor Donald Tuzin, Departamento de Antropología, Universidad de California en San Diego, La Jolla, California 92093" (p. 106), de lo que se deduce que el "Melanesian Archive" se encuentra en San Diego en los Estados Unidos y no en Melanesia. ${ }^{6}$

\section{Conclusiones: John W. Layard en la antropología social británica}

Podemos empezar una evaluación de la vida antropológica de John Layard con las palabras de Evans-Pritchard, quien se llevaba bien con él en Oxford hasta que se pelearan en 1957; decía que "Por supuesto, John Layard está loco, se metió una bala en la cabeza y no hizo diferencia alguna. Debe estar loco" (MacClancy, 1986, p. 65). Sin embargo, creo que el asunto es más complicado y que la cita, tal vez, dice más de Evans-Pritchard que de Layard.

Creo que, en el caso de John Willoughby Layard, hay método en la locura, pero que este método estaba fuera del universo del empirismo británico, tal como ha señalado el mismo Layard. Al respecto, en el primer número de la revista Eranos, en donde Layard publicó en varias ocasiones, Carl Gustav Jung presentó los fundamentos de sus ideas acerca de los ar-

6 Un inmensamente rico y detallado relato de la vida de Layard se puede consultar en Online Archive of California, 1997.

Korsbaek, L. (2021). John Willoughby Layard (1891-1974). Antropólogo y psicoanalista haciendo etnografía en Melanesia antes de Malinowski. Iberoforum. Revista de Ciencias Sociales, Nueva Época, 1(2), 1-28, Artículos, e000190. 
quetipos, la principal herramienta teórica y metodológica del psicoanálisis de Jung y del análisis de Layard.

Acerca de lo inconsciente, dice Jung (2004/1933) que "inicialmente fue una designación para el estado de los contenidos mentales olvidados o reprimidos" (p. 9); luego elabora:

es según ese enfoque, de naturaleza exclusivamente personal, aunque el mismo Freud había visto ya el carácter arcaico-mitológico del inconsciente. Hay un estrato relativamente superficial del inconsciente que es, sin ninguna duda, personal. Lo llamamos inconsciente personal. Pero ese estrato descansa sobre otro más profundo que no se origina en la experiencia, no es una adquisición personal, sino que es innato: el inconsciente colectivo. He elegido la expresión colectivo porque este inconsciente no es de naturaleza individual sino universal, es decir, que en contraste con la psique individual tiene contenidos y modos de comportamiento que son, cum grano salis, los mismos en todas partes y en todos los individuos [...] Los contenidos del inconsciente son en lo fundamental los llamados complejos que constituyen la intimidad de la vida anímica. En cambio, a los contenidos del inconsciente colectivo los denominamos arquetipos. (Jung, 2004/1933, p. 10)

Creo que esta cita puede servir para ilustrar la distancia que obra entre la antropología de Layard y el empirismo británico.

Es cierto que con Evans-Pritchard, Meyer Fortes, Raymond Firth, S. F. Nadel, Mary Douglas, Audrey Richards y Monica Hunter, ${ }^{7}$ más Max Gluckman y sus alumnos de la Escuela de Manchester, nació la antropología social británica moderna; ésta sería desarrollada en las colonias británicas en África (Korsbaek, 2016a), pero es importante resaltar que todo este proceso tuvo una incubación en Melanesia con el trabajo de campo de los antecesores de Malinowski; de esos antecesores, se pondera la contribución de Layard.

Salta a la vista que, en su vida, Layard hizo solamente un trabajo de campo, de aproximadamente un año, en Atchin y las demás islas en Mela-

7 La gran pregunta es: ¿cuándo se dan cuenta los antropólogos sociales británicos que una cosa - observable - es la organización social y que otra cosa - no observable- es la estructura social?; un descubrimiento que hacen los mencionados alumnos de los dos demiurgos (véase al respecto Korsbaek, 2019).

Korsbaek, L. (2021). John Willoughby Layard (1891-1974). Antropólogo y psicoanalista haciendo etnografía en Melanesia antes de Malinowski. Iberoforum. Revista de Ciencias Sociales, Nueva Época, 1(2), 1-28, Artículos, e000190. 
nesia, pero el acervo de información que recuperó en aquella única ocasión es impresionante: "es una de las tragedias de la antropología británica que Layard nunca elaboró su material de Atchin en forma monográfica" (Langham, 1974, p. 238). Quisiera presentar dos sospechas mías al respecto.

Por un lado, pienso que John W. Layard es el seguidor de Rivers que más fielmente y con más argumentos ha defendido la posición de su maestro, misma que fue estigmatizada en la antropología británica a partir de su conversión al difusionismo con su discurso de Plymouth en 1911. Con esta marginación de Rivers se eliminó prácticamente la influencia de la Universidad de Cambridge en la antropología y surgió un vacío que sería ocupado por la London School of Economics, bajo el liderazgo de Malinowski, sobre todo, después de la publicación en 1931 de su análisis de los sistemas australianos de parentesco y su posterior nombramiento a la cátedra en la Universidad de Oxford.

Por otro lado, pienso que, si no fuera por la marginación de Layard en la antropología británica - que, entre otras consecuencias, resultó en la publicación de Stone Men of Malekula en una fecha tan tardía como 1942 (el mismo año que falleció Malinowski y unos años antes de la muerte de Radcliffe-Brown)-, el escenario de la antropología británica podría haber sido diferente, y que las figuras de Malinowski y de Radcliffe-Brown no habrían sido tan dominantes sin la presencia de Layard. El mundo antropológico no habría leído tanto a Los Argonautas del Pacífico Occidental y The Andaman Islanders; habría leído más a Stone Men of Malekula, aunque fuera solamente el primer volumen.

John Layard - más que la mayor parte de la gente que hizo trabajo de campo en el Pacífico en los años cercanos a la Primera Guerra Mundial- y su etnografía incluyeron un nuevo factor: la memoria, pues su obra principal fue escrita más de veinte años después de su estancia en el campo; en este aspecto, supera a Edward Burnett Tylor, quien visitó Cuba y México en 1856 y publicó su libro de viaje Anahuac cinco años más tarde, y se asemeja a Marcel Proust que, como Malinowski, padecía asma y tuvo que aislarse en un departamento forrado de corcho, en donde escribió sus novelas etnográficas apoyándose en su memoria. 


\section{Referencias bibliográficas}

Deacon, A. B. (1934). Malekula. A vanishing people in the New Hebrides. Routledge \& Kegan Paul.

Dunn, J. A. (1974). Review of Malekula. A vanishing people in the New Hebrides. American Anthropologist, 76(4), 910-911. http://www.jstor.org/ stable/674358

Fison, L. y Howitt, A. W. (1880). Kamilaroi and Kurnai. Group marriage and relationship. Osterhout.

Geismar, H. (2009). Stone Men of Malekula on Malekula. An Ethnography of an Ethnography. Ethnos, 74(2), 199-228.

Gell, A. (1998). Arte y agencia. Una teoría antropológica. S. B. Editorial.

Jung, C. G. (2004). Sobre los arquetipos del inconsciente colectivo. En C. G. Jung, W. F. Otto, H. Zimmer, P. Hadot y J. Layard, Hombre y sentido. Círculo Eranos III. (pp. 9-45). Anthropos. (Trabajo original publicado en 1933).

Korsbaek, L. (ed.). (1996). Introducción al sistema de cargos. Universidad Autónoma del Estado de México.

Korsbaek, L. (ed.) (2016). La prehistoria de la Escuela de Manchester: El Instituto Rhodes-Livingstone en el centro-sur de África. Estudios de Asia y África, (159), 199-236.

Korsbaek, L. (2019). El estructuralismo británico pierde su inocencia. Dimensión Antropólógica, 26(77), 32-71. https://www.dimensionantropologica.inah.gob. $\mathrm{mx} / \mathrm{p}=15378$

Kuper, A. J. (1989). Ortodoxia y tabú. Apuntes críticos sobre la teoría antropológica. Universidad Autónoma de Barcelona.

Langham, I. (1974). Obituary: John Willoughby Layard. Oceania, 45(3), 237-239. Langham, I. (1981). The building of British Social Anthropology. D. Reidel.

Layard, J. W. (1928). Degree-taking rites in South West Bay, Malekula. Journal of the Royal Anthropological Society, 58, 139-223.

Layard, J. W. (1930). Malekula: Flying tricksters, gods, ghosts and epileptics. Journal of the Royal Anthropological Institute, 60, 501-524.

Layard, J. W. (1935). The labyrinths in the Megalithic areas of Malekula, the Deccan, Scandinavia and Scotland, with special reference to the Malekulan geometric drawings collected by Deacon, Man, 35, 13.

Layard, J. W. (1936a). Atchin twenty years ago. The Geographical Journal, 88(4), 342-351.

Layard, J. W. (1936b). Maze dances and the ritual of the labyrinth in Malekula. 
Folklore, 47, 123-170.

Layard, J. W. (1937a). Der mythos der Totenfahrt auf Malekula. Eranos, 5, 241-291.

Layard, J. W. (1937b). Labyrinth ritual in South India: Threshold and tattoo design, Folklore, 48(2), 115-182.

Layard, J. W. (1942). Stone Men of Malekula. Chatto \& Windus.

Layard, J. W. (1944a). Song and dance in Malekula. Man, 44, 121-122.

Layard, J. W. (1944b). Primitive kinship as mirrored in the psychological structure of modern man. British Journal of Medical Psychology, 20, 118-134.

Layard, J. W. (1945). The Incest Taboo and the Virgin Archetype. Eranos, 12, 253-307.

Layard, J. W. (1951). The Pilgrimage to Oba. An Atchin sex-initiation rite. En Südseestudien, Gedenkschrift zur Erinnerung an Felix Speiser (pp. 331-357). Museum für Völkerkunde.

Layard, J. W. (1956). Identification with the sacrificial animal. Eranos, 24, 341-406.

Layard, J. W. (1959). Homo-eroticism in primitive society as a function of the Self. Journal of Analytical Psychology, 4, 101-114.

Layard, J. W. (2004). La construcción del hombre en Malekula. En C. G. Jung, W. F. Otto, H. Zimmer, P. Hadot y J. Layard, Hombre y sentido. Círculo Eranos III (pp. 139-195). Anthropos. (Trabajo original publicado en 1948).

Layard, J. W. (s. f.). Study of a Failure [Manuscrito].

Layard, J. W. y Haidy, G. (2005). Slit drums on Atchin. Anthropology and Aesthetics, 47, 191-206.

MacClancy, J. (1986). Unconventional character and disciplinary convention: John Layard. Jungian and Anthropologist. En George W. Stocking (ed.), Malinowski, Rivers, Benedict and Others. Essays on culture and personality (pp. 50-71). University of Wisconsin Press.

Montagu, A. (1944). Review of Stone Men of Malekula. Isis, 35(1), 43-44. http:// www.jstor.org/stable/330700

Online Archive of California (1997). John Willoughby Layard Papers. https:// oac.cdlib.org/findaid/ark:/13030/tf058003wp/entire_text/

"PIC Newsletter", Pacific Information Centre, University of the South Pacific (1990). Layard Papers. Pacific Arts, (1-2), 106. http://www.jstor.org/ stable/23411942 
Rivers, W. H. R. (1914). The History of Melanesia, I-II. Cambridge University Press.

Rivers, W. H. R. (ed.). (1922). Essays on the depopulation of Melanesia. Cambridge University Press.

Spencer, W. B. y Gillen, F. J. (1899). The native tribes of Central Australia.

Spencer, W. B. y Gillen, F. J. (1904). The Northern tribes of Central Australia.

Withers, C. W. J. (2010). A Study of the British Association for the Advancement of Science. Manchester University Press. 